\title{
La elección del tratamiento selvícola y los obstáculos para aplicarlo
}

\author{
Rafael Serrada Hierro \\ SECF. \\ *Autor para correspondencia: rafaelserrada@telefonica.net
}

\section{Introducción y justificación}

Al gestor de montes o gestor forestal, protagonista activo y destinatario de las materias que se contienen en esta Mesa Temática del 7CFE, le corresponde, como actividad más habitual, elegir un tratamiento selvícola para cada uno de los rodales a su cargo. La información para llevar a cabo esta importante y delicada tarea la encuentra el protagonista, de modo central, en las propuestas de la Selvicultura General y en las directrices y experiencias que la Selvicultura Aplicada viene aportando de modo continuo.

Conviene aclarar antes de entrar en materia que, en Selvicultura General, los tratamientos selvícolas se clasifican en relación con su objetivo, como tratamientos de regeneración o tratamientos de mejora. Los tratamientos de regeneración tienen una trascendencia y dificultad mayor que los otros. Sin embargo, una simplificación inadmisible se ha extendido recientemente, incluso en normativas oficiales, por la cual el término tratamiento selvícola se reduce, errónea y exclusivamente, a los tratamientos de mejora.

La mayor parte de los gestores forestales a los que me refiero, y a los que se dirige el presente trabajo, pueden estar incardinados en alguno de los siguientes grupos.

El primer grupo a mencionar, y más numeroso, es el formado por los técnicos de las Administraciones Forestales y empresas públicas, ambas de diferente naturaleza, que tienen a su cargo montes públicos en los que diseñan tratamientos o planificaciones, o en los que aplican planificaciones realizadas por terceras personas. Por otra parte, un importante papel de parte de los gestores de este grupo es autorizar la aplicación de tratamientos en montes privados, tras solicitud de los propietarios y super- 
visando su ejecución. Los componentes de este grupo se encuentran, para desarrollar sus funciones, muy condicionados por una prolija y no siempre acertada normativa administrativa.

Un segundo grupo de gestores, con titulación técnica o no, es el que tiene a su cargo montes privados, bien propietarios forestales, bien gestores en empresas de servicios, bien profesionales en ejercicio libre, quienes, normalmente estimulados por convocatorias de subvenciones al efecto, aplican mejoras en los rodales a su cargo. La normativa sobre subvenciones, aparte de prolija y no siempre acertada al igual que la normativa administrativa que también les es de aplicación, padece el grave defecto de no ser continua en el tiempo, tanto en cantidad de financiación como en calidad técnica. Este grupo también aplica tratamientos para obtener aprovechamientos.

Finalmente, un tercer grupo es el de los gestores forestales en potencia, es decir los alumnos de las Escuelas de Ingeniería Forestal (también de otros centros con otros nombres más o menos equívocos). Son aquellos gestores que, en fase de formación, integrarán en el futuro los dos grupos mencionados anteriormente. Además de la normativa administrativa mencionada, suelen padecer en su formación una cierta desconexión entre las materias académicas que resultan necesarias, que no es únicamente la Selvicultura, para adquirir la capacidad global de gestionar montes. Por otra parte, el enfoque de la impartición de estas materias académicas no siempre está orientado a una aplicabilidad práctica. Esta pérdida de aplicabilidad práctica está conduciendo a un alejamiento de la ingeniería forestal y a una falta de capacidad en el alumnado de abordar decisiones y actividades que resultan, al menos, tan necesarias para la sociedad como la capacidad de abordar y publicar investigaciones.

Seguramente existirá algún tipo de gestor forestal que no pueda ser clasificado en alguno de los grupos que he mencionado. En su caso, pido disculpas y solicito rectificación.

Visto quiénes son los destinatarios de este texto, lo cual es importante para realizarlo y entenderlo, cabe preguntarse por las fuentes de conocimiento para fundamentar correctos criterios de elección del tratamiento selvícola. Como en otros campos, la fuente es la investigación científica en materia forestal. Esta investigación se desarrolla en Centros oficiales y en Universidades y se divulga a través de textos académicos, de comunicaciones a Congresos y de artículos en revistas especializadas, por los investigadores en la materia.

La Selvicultura General propone, globalmente, modelos de tratamientos selvícolas, de mejora y de regeneración. Esta ponencia es una adaptación del último capítulo del texto Apuntes de Selvicultura General (Serrada, 2011). Sobre el contenido y estado de la Selvicultura en España y sus tendencias, remito al lector a la presentación de la obra Compendio de Selvicultura Aplicada en España, obra coordinada por los profesores Montero, Reque y yo mismo en 2008 (Serrada et al., 2008).

Las directrices y experiencias en materia de Selvicultura Aplicada, elaboradas en su mayoría por los mencionados protagonistas relacionados con la actividad forestal que son los investigadores, son los trabajos recogidos, con continuidad en este y anteriores Congresos Forestales Españoles. Por tanto, la consulta de las Actas de los 
CFE publicadas por la SECF es una fuente de información ineludible en la tarea que nos ocupa.

Presentada la tarea y sus protagonistas se resalta la necesidad de conectar la experiencia y la ciencia, la teoría y la práctica, necesidad ya identificada desde hace mucho tiempo (Cotta, 1816), y que resulta ser el objetivo principal, según mi criterio, del presente 7CFE y la conveniencia de analizar el estado de esta conexión, y comprobar los obstáculos que existen y sus tendencias.

La presente Ponencia pretende indicar el modo o proceso en que debe ser elegido el tratamiento de una masa forestal concreta por parte de su gestor, a la vez que en cada fase del proceso se tratará de indicar cuáles son los obstáculos y dificultades que más frecuentemente se manifiestan actualmente en España para que dicho tratamiento, con la calidad debida, pueda ser aplicado, en la forma y urgencia en que ha sido prescrito.

Queda justificado el título y el contenido de la presente exposición, la conexión entre la ciencia y su aplicación práctica y las dificultades para aplicar una correcta práctica selvícola.

En paralelismo con la Medicina, al igual que hizo Cotta (1816), cuando nos enfrentamos a un rodal concreto hay que realizar, sucesivamente, un análisis, un diagnóstico, un pronóstico y, en base a lo observado y deducido en los tres pasos anteriores, proponer y aplicar un tratamiento.

La presente ponencia se centra en el caso de los montes españoles por lo que, inicialmente y a modo de análisis general, se resumen y comentan las características más notables, desde el punto de vista selvícola, de nuestros montes. Posteriormente se aborda el proceso (y dificultades para aplicarlo) de la elección del tratamiento selvícola.

\section{Características de los montes españoles}

Los montes españoles, desde el punto de vista selvícola y a efectos de proponer tratamientos, planteando el análisis de una forma muy genérica, se caracterizan por:

- Variabilidad.- Existe en los montes españoles una alta variabilidad de condiciones estacionales, de composiciones específicas y de formas de masa, incluso entre dos rodales muy próximos. Esta variabilidad de masas se deriva de: la variabilidad climática del territorio español y de la variabilidad meteorológica interanual que le caracteriza; la variabilidad fisiográfica del territorio; y de la variabilidad de las litofacies y de los estados de degradación de los suelos.

Por otra parte, el origen de las cubiertas forestales, la evolución de sus condiciones de uso y de tratamiento que ha experimentado a lo largo de su historia, y la incidencia sobre ellas de perturbaciones (incendios, aludes,...), de régimen más o menos periódico o esporádico, de carácter natural o antrópico, dan lugar a una variabilidad añadida. 
Esta característica implica la necesidad de estudiar y describir con detalle cada rodal particular, huyendo de las generalizaciones. Se puede afirmar, figuradamente, que la práctica de la selvicultura en España es sastrería a medida. Lo que resulta adecuado en un rodal puede dejar de serlo en otro colindante, aun de la misma especie, que ha tenido un tratamiento anterior, o ha sufrido un proceso de degradación, diferente.

La variabilidad afecta también a la fijación de producciones preferentes. Todo ello hace de imposible viabilidad la redacción y la utilización práctica de manuales de selvicultura aplicada a regiones concretas en la forma como se procede en algunos países como Francia o Canadá (Racey et al., 1989), donde la variabilidad espacial de las condiciones estacionales es mucho menor.

Dada la alta variabilidad de las masas de una misma especie, resulta adecuado, para investigar efectos y recomendar tratamientos, elaborar claves tipológicas con base dasométrica que identifiquen diferentes tipos de masa cuya necesidad y tipo de tratamiento puede y debe ser diferente.

Es decir, para cada rodal en que se trabaja, bien para elegir tratamiento, bien para diseñar experimentos, bien para divulgar resultados de los mismos, hay que referirse a las 5 E de la Selvicultura: Estación; Especies; Estructura; Edad y Espesura. Esto, junto con la referencia al origen de la masa y al origen de los pies, completa la necesaria descripción previa.

Otra peculiaridad de las masas forestales españolas, a tener en cuenta en este sentido, es que la capacidad de brotación de cepa y/o raíz de las especies que presentan esta propiedad es muy alta, lo que condiciona las cortas de mejora o de conversión.

- Espesuras defectivas o excesivas.- La aplicación histórica de prácticas anticulturales, la aplicación extendida del monte claro o adehesado en beneficio de determinadas producciones directas, y las dificultades climáticas, como causas más frecuentes, dan como resultado que una gran parte de las masas forestales españolas presenten una espesura defectiva respecto de sus funciones de producción de madera, de protección hidrológica, de mejora del suelo y de mantenimiento de la vida silvestre. Otra situación de masas naturales que presentan espesuras defectivas son aquellas que se están manifestando como consecuencia de procesos de regeneración natural tras abandono agrícola y ganadero o procesos de colonización (Serrada, 2003).

Esto trae como consecuencia crecimientos maderables inferiores a los potenciales y obliga, muy frecuentemente, a aplicar estrategias de capitalización que incluyen el favorecimiento de la regeneración natural y planes de corta que extraigan menos volumen que el crecimiento real para aumentar las existencias y la posibilidad futura.

En otro extremo, como consecuencia de cambios importantes en la apreciación económica de algunas producciones directas y de la falta de inversión en trabajos de mejora por parte de las Administraciones Públicas, aparecen con frecuencia masas cuya espesura es excesiva, tanto para servir con máxima eficacia a sus funciones, 
como para asegurar su estabilidad biológica. Se encuentran en este caso muchos montes bajos y muchas masas artificiales protectoras. Esta segunda situación demanda una amplia aplicación de tratamientos de mejora o de conversión.

- Vulnerabilidad.- Las masas forestales españolas son muy vulnerables a una amplia diversidad de factores bióticos y abióticos. Se comprueba esta afirmación repasando los aportaciones que numerosos autores hacen a las Mesas Temáticas 6 (Incendios) y 7 (Sanidad) de este 7CFE y de los anteriores. En estas comprobaciones también se puede constatar que la vulnerabilidad cambia en el tiempo y se acentúa por razón del cambio global (Serrada et al.,2011).

Especialmente el riesgo de incendio se extiende a todo el territorio. Todas las actuaciones selvícolas, por tanto, pueden y deben tener un alto contenido preventivo frente a enfermedades, plagas e incendios.

- Multifuncionalidad.- En relación con las capacidades productivas de los montes españoles, se puede generalizar afirmando que son obligadamente multifuncionales.

La larga tradición de la actividad ganadera en nuestros montes, presente en la actualidad y con presumible y deseable continuidad en el futuro, plantea, por una parte, conflictos sociales y necesidad de regulación, y por otra parte la posibilidad de contar con una utilidad directa que hay que favorecer en la medida de lo posible.

La rentabilidad de las producciones directas de muchos montes españoles es en la actualidad de muy escaso valor monetario. Esta carencia se acentúa, a efectos de mantener una inversión adecuada en los mismos, por el hecho de que la mayor parte de la superficie forestal es de propiedad privada y tiene una estructura minifundista.

Sin embargo, la prestación de servicios por parte de la superficie forestal, especialmente los relacionados con la protección hidrológica y con la biodiversidad, es de gran interés. Estos comentarios implican que la mayor parte de las inversiones en trabajos forestales deben tener el carácter de mejora, con beneficio preferente para el conjunto de la sociedad y que por tanto deben ser financiados por fondos públicos. En este sentido, ver el lema de este 7CFE. La venta de productos maderables obtenidos en estas tareas puede y debe ayudar a financiar las mejoras.

Como comentario resumen de lo todo anterior, de cara a la práctica de la selvicultura en España, se concluye en la necesidad de inventariar adecuadamente cada rodal para aplicar sobre él un tratamiento, bien de mejora, bien de regeneración, que, orientado por la selvicultura general, resulta ser único y no generalizable

\section{Proceso para la elección del tratamiento.}

El proceso que ayuda a elegir el tratamiento más adecuado en cada caso se resume a continuación, indicando una serie de pasos que es necesario cumplimentar de 
forma ordenada. En cada paso se apuntan los obstáculos o dificultades que el gestor debe superar, con mayor frecuencia, para conseguir aplicar el tratamiento prescrito. Los pasos 1 a 4 se corresponden con la analítica, los pasos 5 y 6 con el diagnóstico, los pasos 7 y 8 con el pronóstico y, finalmente, los pasos 9 a 14 con el tratamiento:

$1^{\circ}$.- Estudio del Estado Legal y Socioeconómico. Puede considerarse como una cuestión previa y basada en aspectos no selvícolas. Sin embargo, de este estudio se derivan tres aspectos trascendentes relacionados con la planificación: fijación de la producción preferente del monte teniendo en cuenta las intenciones de los propietarios y del conjunto de la sociedad; indicación de algunas importantes restricciones normativas; y el papel del monte en relación con el empleo.

Las dificultades u obstáculos que se encuentra el gestor forestal en relación con este primer paso del proceso son de gran calado y se derivan de la percepción que el conjunto de la sociedad, cada vez más urbanita, tiene del funcionamiento y gestión de los ecosistemas en general y de los forestales en particular. Esta concepción supera la escala de monte o rodal y afecta al derecho de los propietarios, a la fijación de objetivos preferentes y a la negación del papel que los montes deben tener en el desarrollo rural.

La percepción del conjunto de la sociedad sobre la naturaleza y sus componentes padece de un equivocado antropomorfismo o humanización que consiste en atribuir características y cualidades humanas a los animales de otras especies, y a objetos o procesos naturales. Un antropomorfismo sensiblero se instala en la sociedad moderna a partir de la película Bambi de Walt Disney, estrenada en 1942. En esta película, los elementos poseedores de belleza, aspecto humano, bondad, palabra y sentimientos son los animales, mientras que los humanos son egoístas, matan y destruyen el bosque, todo ello sin ninguna expresión oral.

Una consecuencia de estos planteamientos se puede resumir en el siguiente silogismo:

- Premisa mayor: La naturaleza es magnífica y debe ser conservada y protegida.

- Premisa menor: El hombre, por su mera presencia, es malo para la naturaleza.

- Conclusión: Todo lo que se haga para perjudicar al hombre es bueno para la naturaleza.

Evidentemente la premisa mayor es cierta, pero la premisa menor es totalmente errónea lo que da lugar a una conclusión de muy negativas consecuencias.

La primera y negativa consecuencia es que quien reconoce como cierto el silogismo, al ser parte constituyente del género humano, entra en una contradicción vital o en un trastorno de identidad disiociativo que únicamente puede resolver por la vía del maniqueísmo: yo y los míos somos buenos, lo malos son los otros, hay que perseguir a los otros y ese es el único medio de salvar la naturaleza.

Este maniqueísmo se asienta, en la sociedad española, en los años 80 del siglo pa- 
sado en los movimientos ecologistas, incluso se traslada a las especies vegetales arbóreas y a las masas forestales, contribuye a la simplificación de los mensajes en los medios de comunicación y, finalmente, en la primera década del presente siglo se instala en gran parte de la normativa administrativa que regula la gestión de la naturaleza.

En la actualidad, una consecuencia de esta concepción de la naturaleza y su gestión es el movimiento animalista, en muchos aspectos y manifestaciones radicalizado.

Para el gestor forestal, en los estudios y propuestas relacionados con este primer paso del proceso, los obstáculos relacionados con el antropomorfismo, el maniqueísmo, la simplificación y el animalismo se refieren a la negación del derecho de los propietarios de los montes a su gestión (aunque ésta se realice dentro de lo que la ortodoxa técnica forestal prescribe), bien en la opinión pública, bien en las decisiones administrativas, como si el obtener rentas de los montes fuese malo en sí mismo. Todo ello sin tener en cuenta la contribución de estas actividades al empleo y al desarrollo rural. Este es uno de los factores que contribuyen al despoblamiento rural.

Se pueden buscar muchos ejemplos de cómo este planteamiento ha inundado el repertorio actual de normas administrativas, especialmente en los PORN y los PRUG de espacios naturales protegidos, que suelen poner un gran empeño en expulsar de estos espacios cualquier actividad humana. Sirva como ejemplo de lo afirmado que la Ley 30/2014, de 3 de diciembre, de Parques Nacionales dispone, en su artículo 7.3, que se considera actividad incompatible en estos espacios la tala con fines comerciales. Es decir, las cortas no se clasifican como de mejora o de regeneración, simplemente no se puede vender la madera para que sean compatibles. Por otra parte, al legislador no le parece mal que se venda la carne de las vacas del PN de los Picos de Europa u otros. Otro ejemplo con menor trascendencia territorial y temporal es la Orden de 26/12/2013, de la Consejería de Agricultura, por la que se establecen las bases reguladoras de las ayudas para la elaboración de revisiones de instrumentos de gestión forestal conforme a los postulados de la Gestión Forestal Sostenible y para la ejecución de tratamientos selvícolas en el marco del programa de desarrollo rural de Castilla-La Mancha 2007-2013 y se efectúa su convocatoria para el año 2014, que establece en su Anexo III que no se consideran subvencionables aquellos trabajos de los que se obtengan productos que posteriormente sean objeto de comercialización. Fue, afortunadamente, derogada en 2016.

Bien, consideremos, para seguir con la descripción del proceso de elección del tratamiento en el monte o rodal en cuestión, que la propiedad en concordancia con una correcta normativa administrativa y las características del lugar, ha fijado una producción preferente concreta y señalado todas las demás compatibles con ella en aplicación de la multifuncionalidad.

$2^{\circ}$.- Estudio de la estación. El estudio climático, fisiográfico y edafológico completo es la base para dos importantes pasos posteriores: el diagnóstico de posible marginalidad ecológica, actual y futura, de la masa forestal actual, y 
sobre la calidad de estación; y la división del monte en rodales. El estudio edafológico resulta especialmente necesario para apreciar posibilidades de mejora en las propiedades físicas y químicas y valorar la marginalidad.

Es muy importante la identificación de posibles factores ecológicos limitantes para las masas presentes. Se recomienda aplicar, en estudios de marginalidad actual y futura incorporando hipótesis de cambio climático, las metodologías basadas en los estudios de autoecología paramétrica de las especies forestales españolas, que requieren estudios edafológicos, en el modo en que quedan descritas en Gómez-Sanz et al. (2017a y 2017b). Estos trabajos están basados en las numerosas publicaciones sobre autoecología paramétrica de las especies forestales arbóreas españolas desarrollados por los profesores Gandullo y Sanchez-Palomares, que contienen una inestimable información para estos fines.

Resulta desconcertante el escaso uso que se hace de estudios edafológicos, con toma de muestras y analítica adecuada, para los proyectos de ordenación de montes y de repoblaciones forestales, siendo frecuentemente sustituidos por imprecisas aplicaciones de cartografías de suelos en los que no se pueden detectar las limitaciones que las propiedades físicas y químicas imponen en cada rodal concreto. Cabe preguntarse por la conexión entre materias tales como edafología y selvicultura en los planes de estudios universitarios y tratar de mejorarla.

$3^{\circ}$.- Estudio de la vegetación.- La vegetación actual quedará descrita mediante una división en zonas homogéneas, identificadas en primer lugar por la presencia de diferentes formaciones y matizada, dentro de cada formación, por posibles variaciones de composición específica en primer lugar y de espesura en segundo lugar.

La consideración conjunta de este y el anterior punto aportan la base para la posterior división en rodales.

La vegetación potencial puede ser identificada a través de la presencia de especies indicadoras y con la ayuda de cartografía.

Una dificultad notable en este punto del proceso se refiere a la muy extendida, y poco racionalizada, sacralización del concepto de climax. Esta concepción de la vegetación potencial, divulgada por la fitosociología sigmatista, que sin tener en cuenta la influencia de otros factores ecológicos además del clima, ni cuestiones relacionadas con las escalas temporales, abunda en el maniqueismo citado anteriormente y acaba en una injustificada xenofobia vegetal (Gil Sánchez, 2008 y 2009).

$4^{\circ}$.- Inventario.- Con una posible división inventarial previa, basada en el estudio de la vegetación actual, se realiza un inventario forestal que aporte información precisa sobre: composición específica; distribución de edades de los pies, edad que no siempre puede ser determinada y puede ser inferida por el tamaño; estratificación y estructura; origen de los pies; índices de espesura; estado de la regeneración; dinámica de la masa; etc. 
Hay que tener presente que, en cada caso, la toma de datos y el diseño del inventario deben ser propuestos, dentro de las directrices genéricas de esta disciplina, con el fin de satisfacer las necesidades de información que las posibles alternativas de tratamiento particular van a presentar.

También en el diseño del inventario se debe trabajar huyendo de métodos estandarizados, que han venido siendo orientados, preferentemente, a la estimación de volúmenes de madera. Esta estimación de volúmenes maderables y de crecimientos no tiene en la actualidad la trascendencia que tuvo en el pasado por los siguientes motivos: a efectos de control en operaciones comerciales es posible la pesada o cubicación de camiones; las nuevas tecnologías (LIDAR y otras) ofrecen información detallada con menor trabajo de muestreo en campo; existen modelos matemáticos informatizados para realizar cubicaciones y estimaciones de crecimiento en muchas especies.

En este sentido, se han convertido en un obstáculo las Instrucciones de Ordenación de Montes Arbolados vigentes con diferentes ámbitos territoriales que no han sido actualizadas. Estas obsoletas directrices deberían ir siendo sustituidas por las nuevas metodologías que podemos encontrar, por ejemplo, en Reque y Pérez (2011) o en Reque et al. (2013).

$5^{\mathrm{o}}$.- Apeo de rodales.- Este punto tiene una gran trascendencia. Con base en el estudio de la estación, de la vegetación y de su estructura y espesura, aspectos identificados en el inventario, se formula la división de la zona en estudio en unidades elementales sobre las que aplicar un tratamiento homogéneo en toda su extensión.

Se identifican los rodales en los que, en el momento presente, no es necesario aplicar ningún tipo de tratamiento. Más erróneo que aplicar un tratamiento inadecuado en un rodal es proponer y ejecutar trabajos allí donde no son necesarios, dada la enorme escasez de disponibilidad presupuestaria en el sector forestal. Esta escasez se deriva de la ausente voluntad política en este ámbito y de la invisibilidad a las que se ha condenado a los montes, situaciones que no son ajenas al maniqueismo y simplificación que se han mencionado anteriormente.

En el resto de los rodales se indica y justifica lo que es necesario hacer. El tamaño de los rodales será lo suficientemente grande como para permitir una ejecución razonable de las tareas selvícolas, y lo suficientemente pequeño como para no incluir bajo la misma linde zonas o situaciones heterogéneas.

$6^{\circ}$.- Estudio de los caracteres culturales de la especie o especies presentes.Deben ser recordados en este punto los caracteres culturales de la especie o especies presentes en cada rodal (Bravo-Oviedo y Montero, 2008).

La estación aportará diagnóstico sobre posible marginalidad estacional actual o futura. Este diagnóstico se perfecciona aplicando la metodología de Gómez-Sanz et al. (2017a y 2017b), citada anteriormente. 
El temperamento informa sobre las condiciones de espesura para conseguir la regeneración natural.

$7^{\circ}$.- Análisis de los antecedentes selvícolas.- En este punto se analizan de forma ordenada toda clase de fuentes que puedan aportar información y experiencia sobre el caso particular, bien relacionadas con la especie o especies, bien con la función preferente, y todo ello en un contexto geográfico-ecológico adecuado.

Es preciso en, este punto de análisis de antecedentes, vacunarse contra lo que podemos denominar el síndrome del descubridor. Resulta frecuente que cuando un gestor o un legislador ve por primera vez un rodal que le deslumbra por su novedad, complejidad y belleza, lo considera como algo propio, decide que debe ser conservado tal cual lo ha descubierto sin entender que es algo dinámico y desprecia e ignora los procesos, actividades y personas que han actuado con anterioridad para gestionar lo que ahora se le aparece. Sus muy frecuentes propuestas de congelación para la conservación resultan erróneas.

Las fuentes para completar el análisis, de diferente condición y naturaleza, son las siguientes:

- Bibliografía referente a la especie y forma de masa, que en todo caso hay que interpretar en relación con las condiciones estacionales de las zonas donde se han realizado las experiencias y/o propuestas.

Un obstáculo que puede encontrar el gestor, en caso de abordar esta tarea de recopilación bibliográfica, es que una gran parte de la producción científica, según las directrices impuestas desde hace tiempo para la valoración (exclusiva) de quienes la producen, ha de ser publicada en revistas llamadas de impacto. El acceso a estas publicaciones no resulta fácil para el gestor, por motivos económicos y de idioma (Serrada, 2005). Por otra parte, esta paper-adicción determina que son preferentes las líneas de investigación que tienen mayor probabilidad de ser publicadas frente a aquellas que pueden resultar más necesarias en el contexto real del trabajo en el monte.

Una vía para superar este obstáculo de la difícil divulgación de investigaciones y experiencias en el sector forestal ha venido siendo la celebración de los Congresos Forestales Españoles y la disposición del eficiente y completo buscador de textos de la página web de la SECF. Esperemos que, a pesar de cierta deriva que se viene observando, esta función no sea abandonada en el presente 7CFE y siguientes.

- Experiencias y trabajos que están en curso sobre montes cercanos o semejantes en cuanto a suelo y vuelo. El obstáculo en este sentido se refiere a dos cuestiones: la falta de tiempo para viajar y ver otros trabajos; la existencia de nuevas fronteras administrativas que limitan, cada vez más, el desplazamiento de los funcionarios. 
- Estudio de toda clase de antecedentes documentales sobre el monte en cuestión. Sobre la mayor parte de los montes españoles existen referencias documentales más o menos antiguas, que pueden aportar mucha luz sobre la explicación del estado actual del monte y, por tanto, sobre su dinámica.

El obstáculo en este punto es que la localización de documentos, dada la frecuente desidia administrativa, no siempre resulte fácil. Es necesario hacer un continuo esfuerzo en este sentido.

- Antecedentes orales, pues los antiguos gestores, técnicos y guardas, de los montes conocen muchos detalles sobre la historia y los trabajos en cada monte que no necesariamente han quedado registrados en documentos. Conseguir captar esta información, lo que requiere un propósito y una dedicación adecuados, es muy importante para completar la información y para evitar diagnósticos subjetivos. El obstáculo suele ser la dificultad en encontrar y obtener la colaboración de estas personas.

- La observación detallada de hechos y procesos en el propio monte. La falta de datos y de información que muchas veces se hace sentir acerca de tratamientos o sucesos en el monte puede ser suplida por una atenta observación en el propio monte.

Los viejos tocones, las copas deformadas, el hollín sobre los fustes de pies gruesos, la acumulación de piedras o tierra en los arroyos, etc, son parte de un lenguaje que hay que intentar traducir. El monte es el principal maestro de los forestales y los forestales deberían ser los portavoces del monte en relación con sus enseñanzas y necesidades.

$8^{\mathrm{o}}$.- Decisión sobre la forma fundamental y principal de masa.- Hasta este punto se ha dedicado la actividad a recopilar información, siendo la única decisión tomada la relativa a la función preferente de la masa. Conocida la masa en todos sus aspectos, estáticos y dinámicos, y sabiendo cuál es la función principal que debe desempeñar, se está en condiciones de proponer la forma fundamental y principal que mejor se adapte a dicha función y al uso múltiple, y que sea posible por razón de las restricciones específicas y estacionales. En definitiva, se trata de proponer el método de beneficio y el tratamiento de regeneración más adecuados conjugando aspectos ecológicos o selvícolas, sociales y económicos.

A este respecto hay que advertir que los modelos de masa o forma de materializar los tratamientos que la Selvicultura General propone deben ser interpretados como una orientación general. Son estructuras definidas como puntos de referencia dentro de un continuo.

En relación con la descripción de las formas principales de masa, hay que hacer 
notar la relación que existe entre tiempo y espacio, y la importancia del tamaño y forma de las unidades de regeneración, de modo que se comprenda que, en definitiva, lo que distingue a una corta a hecho de una corta por entresaca es el tamaño de la superficie en la que se pretende que se incorporen nuevos pies en un plazo determinado. Sobre el tamaño y forma de la superficie en que se pretende producir la regeneración hay gran variedad de denominaciones, por lo que, aparte de cuestiones relacionadas con la sistemática o denominación de las unidades, hay que centrar la atención en el efecto que la masa adulta provoca sobre el microclima de la zona a regenerar.

Se debe comprender que en el conjunto de cualquier cuartel tiene que haber pies de todas las clases de edad, con mucha mayor proporción de los jóvenes que de los grandes. Los tratamientos generales se diferencian en cómo se reparten estos pies en el espacio.

Las diferencias entre tratamientos se refieren al grado de nivel de cambio ambiental que ocasionan, respecto de la situación inicial. El mínimo grado de alteración está en la entresaca pie a pie y el máximo en las cortas a hecho. Hay que relacionar esta idea con el posible origen natural de las distintas formas de masa y con la estructura y espesura que las definen:

- Las masas regulares pueden tener origen natural en catástrofes y pueden, y deben, mantener espesuras relativamente más altas durante la fase de desarro1lo, por lo que su regeneración inducida, cuando llega a madurez, es brusca y afecta a una espesura inicial mayor que en las masas irregulares, aunque haya sido conducida con cortas de mejora.

- Las masas irregulares pueden tener origen natural en una incorporación continua de pies aprovechando la mortalidad natural de los pies de gran desarrollo y deben, en la gestión selvícola, mantener espesuras relativamente más bajas para mantener la regeneración a la espera en toda la superficie, por lo tanto, las cortas que las mantienen alteran en menor medida una espesura ya de por sí baja.

En todos los tratados de Selvicultura se ha seguido el conocido orden de explicación: cortas a hecho; cortas por aclareo sucesivo uniforme; cortas por entresaca. Tal vez un motivo de esto sea que en este mismo orden se fueron proponiendo históricamente los tratamientos en el desarrollo de la Selvicultura como técnica y ciencia. Otro posible motivo de esta forma de exponer puede ser que, al igual que en Botánica y Zoología se empieza explicando los organismos menos complejos para terminar en los más evolucionados, sea más fácilmente comprensible pasar de esquemas de gestión simples (cortas a hecho) a sistemas complejos (cortas por entresaca).

En principio no es criticable el orden de exposición clásico, pero puede mejorarse la forma en que se exponen las representaciones gráficas de los diferentes tipos de masa que resultan de los tratamientos generales: siempre se hace forma lineal, cuan- 
do se podría mejorar la comprensión haciendo una representación en forma circular. En la figura 1 se expone esta idea, donde queda representado cada tipo de tratamien-

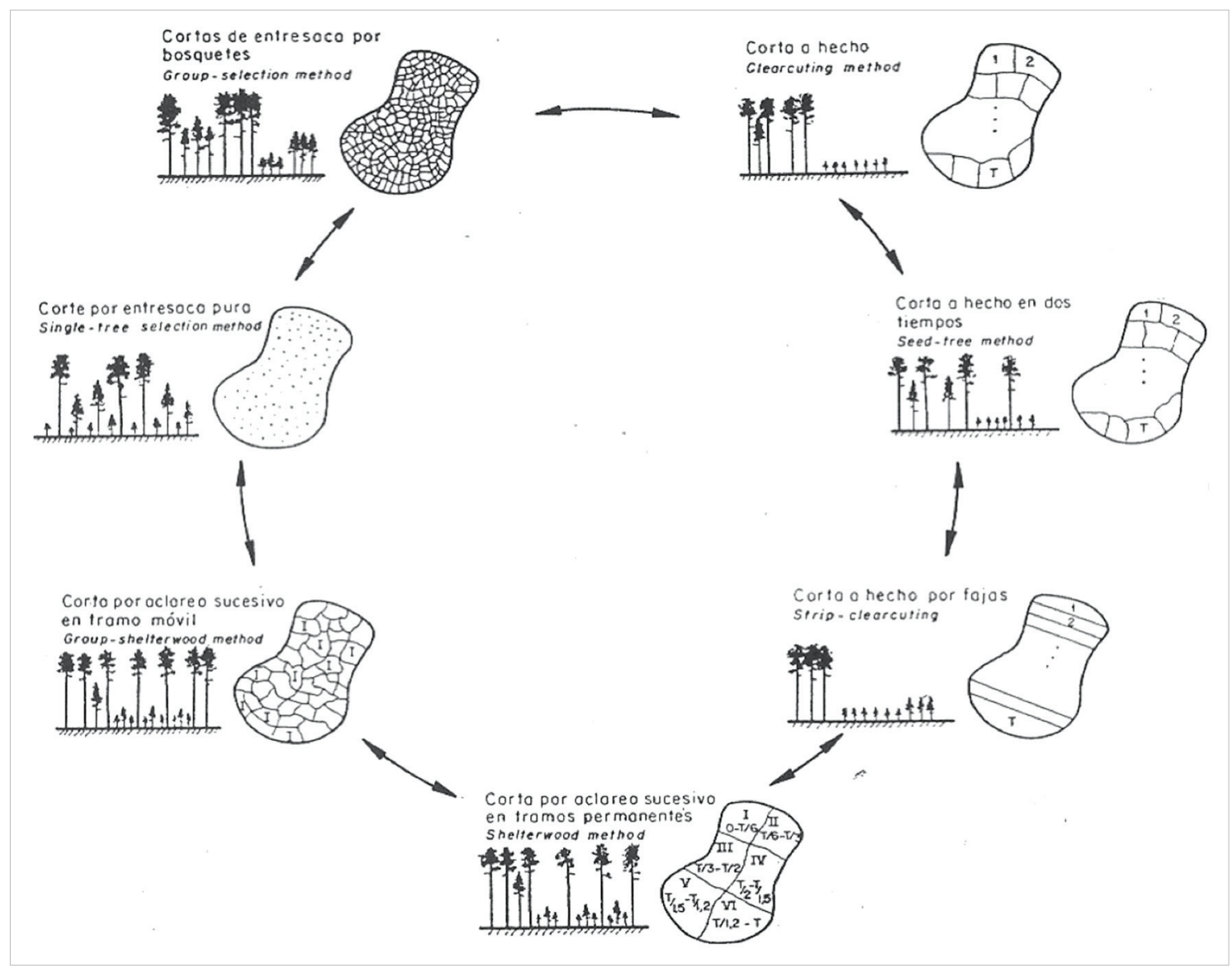

Figura 1. Representación en un esquema circular de los tratamientos generales y de las formas principales de masa propuestos por la selvicultura general. Dentro del mismo cuartel, la superficie de las unidades de regeneración van cambiando de tamaño y el tiempo para completar las mismas también. Según Serrada, Madrigal y González Doncel (1997).

to por un esquema del reparto de edades en el espacio y un pequeño dibujo que trata de representar la masa en estado de regeneración:

Una vez propuesta la forma de masa más adecuada según las directrices anteriores, se actuará con flexibilidad, dentro de una planificación orientada por el tratamiento general correspondiente, y redactada de acuerdo con las Instrucciones de Ordenación de Montes Arbolados que estén en vigor en cada caso, teniendo en cuenta que, según se ha expresado anteriormente, hay que interpretarlas teniendo en cuenta los cambios selvícolas, económicos y sociales que se han producido desde que se redactaron hasta la actualidad.

Un obstáculo en este sentido es dotar de un rigor reglamentario excesivo a las planificaciones que estén en vigor. El que se pueda y deba actuar con flexibilidad no significa que no deba proponerse una planificación, pero el cumplimiento ciego de pla- 
nificaciones propuestas en tiempos anteriores, bajo condiciones y normativas que pueden ser obsoletas, puede resultar negativo.

$9^{\circ}$.- Tratamientos parciales.- En principio hay que hacer una propuesta básica, en relación con la posible aplicación de tratamientos parciales en un rodal que es la siguiente: antes de aplicar un tratamiento parcial hay que haber definido la forma principal y fundamental de masa concordante con su función preferente, aparte de, por supuesto, el objetivo de dicho tratamiento parcial.

El hecho de condicionar a que deba conocerse la estructura y el origen de los pies que deseamos para la masa que estamos tratando para definir la aplicación de desbroces, clareos, claras o podas es totalmente lógico. De otra forma no se pueden fijar con corrección las características de dichos tratamientos parciales.

Sin embargo, y al contrario de lo afirmado, en algunas ocasiones puede estar justificado actuar aunque no se haya fijado el tratamiento general, como por ejemplo: casos de muy alta espesura en rodales de estructura regular, donde se pueden aplicar claras bajas de peso moderado que eviten la desestabilización biológica, aunque no se haya definido en este momento su función preferente; o, en general, tratamientos preventivos de incendios en masas de alta combustibilidad.

Un frecuente obstáculo para realizar adecuadamente estos tratamientos es el que podríamos llamar el síndrome de no sabemos cuánto tiempo vamos a tardar en volver a este rodal. No son excusa para proponer y ejecutar un tratamiento inadecuado los condicionantes administrativos o la frecuente usura presupuestaria. El monte no tiene la culpa de estas cosas y debe recibir el mejor trato posible en todas las situaciones. Dicho de otro modo, las cosas se hacen bien o no se hacen.

Este modo de pensar no solo afecta a decisiones concretas en un rodal concreto, también se han instalado en una normativa administrativa que pretende regular, a través de un boletín oficial, todas las actuaciones de modo simplista en un universo de gran complejidad.

Citamos un par de ejemplos sobre normativa infundada y errónea que se convierte en un obstáculo para la correcta actuación de los gestores.

Como ejemplo de conservadurismo y limitación de actuaciones, en el año 2002, y vigente en la actualidad 15 años después, se publica la Orden de 31-01-2002, de la Consejería de Agricultura y Medio Ambiente de Castilla-La Mancha, por la que se declara a la sabina albar especie de aprovechamiento regulado en la parte de su distribución donde no está considerada especie de interés especial, y se establecen normas técnicas para la regulación de su aprovechamiento. En esta norma, aparte de imponer que las podas de ramas únicamente se ejecuten con hacha, en el artículo 3, literalmente, se establece la siguiente regulación para la corta de pies de sabina albar, de ejemplares vivos o muertos: 4. No se podrá otorgar autorización si concurre alguna de las siguientes circunstancias: g) Cuando el pie que se pretende cortar diste más de 3,2 $m$ de cualquier otro pie a respetar con diámetro igual o superior (densidad media de 1000 pies/ha), salvo en los casos en que se pueda justificar que el área basimétrica a la altura de $130 \mathrm{~cm}$ tras la corta supera los $20 \mathrm{~m}^{2} / \mathrm{ha}$. No es ne- 
cesario resaltar el reglamentismo estéril de esta disposición, máxime cuando en las masas de sabina de la región no se han encontrado rodales con área basimétrica superior a $15 \mathrm{~m}^{2} \mathrm{ha}^{-1}$, eso sí, medida a altura normal.

Un ejemplo de norma errónea que propone actuaciones de intensidad excesiva es la Orden de 01/07/2009, de la Consejería de Agricultura y Desarrollo Rural de Castilla-La Mancha, por la que se establecen las bases reguladoras y el plazo de las convocatorias de las ayudas a la implantación de medidas preventivas de lucha contra incendios forestales en el marco del programa de desarrollo rural de Castilla-La Mancha 2007-2013. En ella, únicamente se subvencionan las Áreas cortafuegos cuando se elimine en su totalidad la vegetación arbórea manteniendo su densidad inferior al $10 \%$ de Fracción de Cabida Cubierta (FCC). No se distingue en relación con la posibilidad de brotación de las especies, con la espesura inicial, con la pendiente, ni con ninguna otra circunstancia. Cualquier gestor que pretenda realizar una selvicultura preventiva razonable debería renunciar a la subvención.

$10^{\circ}$.- Plazo para la aplicación del tratamiento.- El plan de tratamientos se diseña para ser ejecutado durante el tiempo suficiente, largo plazo, como para que pueda producir los resultados buscados. No es correcto apresurarse en selvicultura y buscar resultados a corto plazo.

En selvicultura los éxitos de una acción determinada son difíciles de apreciar, dado el largo plazo que tienen para manifestarse.

Al igual que en la valoración de la eficacia en la extinción de incendios forestales, en la que el modo más adecuado, y difícil, es aplicar la frase del conocido bolero: lo que pudo haber sido y no fue, también en la valoración del resultado de un correcto y necesario tratamiento selvícola, resulta muy difícil valorar el estado de la masa en caso de no haber actuado con acierto en su momento.

También los fracasos tardan en aparecer, salvo flagrante actuación anticultural. Se actúa con cierta impunidad, especialmente en lo relativo a no proceder a actuaciones que puedan considerarse necesarias para la estabilidad o la regeneración de la masa, o al hecho de aplicar cortas de excesivamente baja intensidad o peso.

Un obstáculo frecuente en este sentido es considerar que la respuesta de todas las especies en una comarca ha de ser similar o que una misma especie debe responder uniformemente en todas las comarcas.

$11^{\circ}$.- El aprovechamiento como herramienta de mejora.- En la selvicultura extensiva, aplicable en la mayoría de la superficie forestal arbolada de España, hay que mantener la consideración de que el aprovechamiento, la extracción de productos directos (de tan mala prensa) en términos de rentabilidad financiera, es el mejor medio para la mejora y mantenimiento de la masa. Las cortas son la vez fin y medio. Fin para dar empleo en la comarca y rentabilidad a la propiedad del monte. Medio que garantiza la persistencia y la estabilidad de la masa. Existen notables ejemplos en este sentido en toda nuestra geografía (Cabrera, 2001). 
Los obstáculos en este punto ya se han expuesto en el primer punto del proceso: la sociedad opina de modo general que cortar árboles es muy malo.

$12^{\circ}$.- Regeneración.- Atender a la regeneración es, lógicamente, la base de la persistencia y por tanto completamente trascendente.

Si se formula esta advertencia en este punto es por razón de la constante presencia actual, y suponemos que futura, del pastoreo en los montes. La actividad pastoral tiende a ser excesivamente consentida por el conjunto de la sociedad y de los poderes públicos, sin aceptar las lógicas restricciones que, como toda actividad que se desarrolla en el medio natural, debe tener. Un exponente de esta afirmación es la eliminación del acotado al pastoreo de, al menos, un año que se estableció en la Ley de Montes de 2003 y que ha sido derogada por la reforma de 2015, y que ha dado lugar a un notable incremento de la siniestralidad por incendios forestales en Asturias y Cantabria durante 2016 y 2017.

No se deben interpretar estos comentarios como una propuesta de exclusión de la ganadería extensiva en nuestros montes, al contrario, debería ser potenciada en los lugares, con las especies, en el tiempo y con las cargas más adecuados en relación con los cuatro factores. La ganadería extensiva es un instrumento de potenciación del desarrollo rural y de reducción de la combustibilidad.

Por otra parte, en selvicultura extensiva la regeneración, adecuadamente inventariada, debe tender a ser natural, lo que no debe descartar, en casos de especial retraso, la ayuda a la misma o incluso la regeneración artificial.

$13^{\circ}$.- Regeneración incorporada.- El control de la regeneración conseguida tras las cortas correspondientes debe ser realizado por inventarios sistemáticos y con fijación previa de valores de densidad admisible.

Los pies presentes en las zonas de regeneración y cuya edad está alejada del turno, siempre que muestren vigor, serán incorporados a la nueva masa aunque esto suponga un cierto grado de irregularización.

Hay que liberar los regenerados conseguidos de la competencia de la masa vieja, independientemente de la asignación temporal que la planificación imponga. Hay que aplicar cuidados culturales en los regenerados: desbroces y clareos.

En este sentido, las planificaciones basadas en la extracción de unos volúmenes de madera limitados se convierten en un obstáculo. Lo importante es planificar ordenando superficies, en modo similar como se procede en la entresaca regularizada, para que ningún rodal se quede sin recibir las actuaciones necesarias en plazos superiores a 15 o 20 años, en los que la regeneración a la espera empieza a decaer por exceso de espesura o en los que los bosquetes de masas regulares superan una rotación de claras razonable.

$14^{\circ}$.- Para regenerar, hay que cortar.- Siendo la regeneración el futuro y la estabilidad de la masa, la regeneración se consigue cortando (bien) y por tanto, 
las cortas son necesarias para que las masas mantengan su vigor y continuidad.

Un modo de perjudicar a los bosques, muy extendido en la actualidad, es abandonar todo tipo de cortas. Se puede imputar esta tendencia a dos motivos: no son aparentes los resultados de las inversiones en el sector forestal y por tanto los presupuestos son escasos; y la crítica, actual e infundada, sobre este tipo de actuaciones deja paralizados a los responsables de la gestión, pues nadie critica que no se haga nada. Para comprender el error de la pasividad hay que hacer un gran esfuerzo en el estudio y comprensión de la selvicultura, tanto general como aplicada.

\section{Clases de Selvicultura}

La Selvicultura es una ciencia-técnica que define a la ingeniería forestal. Desde su nacimiento como tal, de la mano de Cotta (1816) hasta nuestros días ha ido cambiando y adaptándose a nuevas demandas sobre los montes y mejorando su capacidad con el desarrollo de otras ciencias y tecnologías. La capacidad del gestor o ingeniero forestal se adquiere, tras el aprendizaje de la Selvicultura General, con el estudio de la Selvicultura Aplicada. Se recomienda, a los miembros de tercer grupo de gestores expuesto anteriormente, continuar el estudio a través del texto de referencia Serrada, Montero y Reque (editores) (2008).

La selvicultura aplicada puede tener dos enfoques principales: Selvicultura Específica y Selvicultura Funcional.

La selvicultura específica contiene directrices, por especies y tipologías de masas, basadas en experiencias controladas, que cada vez son más detalladas y desarrolladas. En este aspecto se desarrolla la mayor parte de la investigación dentro del campo selvícola.

La selvicultura funcional, la que orienta para manejar masas según la función preferente asignada, está, al igual que la selvicultura general, bastante desarrollada. Entre las recomendaciones de la selvicultura funcional se encuentran, por ejemplo: la selvicultura preventiva de incendios; la selvicultura de protección hidrológica; la selvicultura de los espacios naturales protegidos, con función principal paisajística y de mantenimiento de la biodiversidad; la selvicultura para fijación de carbono.

Aunque a la hora de proyectar o de financiar un tratamiento selvícola determinado en un rodal concreto, su objetivo preferente le puedan hacer ser calificado de forma parcial como, por ejemplo, preventivo de incendios o de aprovechamiento energético, nunca hay que olvidar que influirá en otros muchos aspectos y funcionalidades de la masa y que deberá ser propuesto tras un completo análisis, un correcto diagnóstico y ser concordante con el pronóstico esperado. Hacer las cosas sin este estudio previo, por el hecho de que se hace así habitualmente, es un empirismo inaceptable.

Finalmente, hay que hacer mención a la selvicultura urbana, término que aunque en principio pueda parecer contradictorio, es aceptable en la medida en que el arbo- 
lado urbano, parques, plazas y calles, tiene comportamientos y respuestas a la espesura y su variación semejantes al arbolado forestal.

Termino, para estimular el estudio y la investigación aplicada en el campo selvícola, a la vez que pretendo dar confianza al ingeniero forestal, con una frase tomada de Cotta (1816): Apenas es creíble cuanto se puede beneficiar o perjudicar un monte por el modo de tratamiento, y la verdadera ciencia forestal contiene, por consiguiente, muchísimo más de lo que presumen los que solamente conocen lo vulgar de ella.

\section{Bibliografía}

Bravo-Oviedo, A; Montero, G., 2008. Descripción de los caracteres culturales de las principales especies forestales de España. En Serrada, R.; Montero, M. y Reque, J. (editores). 2008. Compendio de Selvicultura Aplicada en España. INIA y FUCOVASA. Madrid. pp. 1.039 a 1.114.

Cabrera, M (ed.), 2001. Actas de la III Reunión sobre evolución de Ordenaciones históricas. Cuadernos de la Sociedad Española de Ciencias Forestales, número 11 - 2001. Sociedad Española de Ciencias Forestales. Grupo de Trabajo de Ordenación de Montes. Almazán, 26 a 28 de junio de 2000.

Cotta, H., 1816. Anweisung zum Waldbau (Consejos de Selvicultura). Traducción por Herbella, E., 1912. "El prólogo de la Selvicultura de Cotta". Revista de Montes. año XXXVI, pág. 317-320. Asociación de Ingenieros de Montes. Madrid.

Gil Sánchez, L., 2009. Una desmedida devoción por los matorrales (o por qué estabilizar el paisaje humanizado). Actas del 5CFE. SECF. Ávila. 44 pp.

Gil Sánchez, L., 2008. Pinares y rodenales. La diversidad que no se ve. Discurso leído el día 23 de septiembre de 2008. Real Academia de Ingeniería, Madrid, 191 pp.

Gómez-Sanz, V.; García Viñas, J.I; Serrada Hierro, R., 2017a. Medio físico y decaimiento de rodales de Pinus halepensis Mill. en la Comunidad Valenciana. Actas del 7CFE. SECF. Plasencia. $12 \mathrm{pp}$.

Gómez-Sanz, V.; García Viñas, J.I; Serrada Hierro, R., 2017b. Medio físico e inestabilidad de masas forestales meso-supramediterráneas en espacios naturales gestionados por el Organismo Autónomo de Parques Nacionales. Actas del 7CFE. SECF. Plasencia. 12 pp.

Racey, G.D.; Whitfield, T.S. \& Sims, R.A., 1989. Northwestern Ontario Forest Ecosystem Interpretations. Forestry Canada. Ministry of Natural Resources. Ontario.

Reque, J; Pérez, R., 2011. Del Monte al Rodal. Manual SIG de Inventario Forestal. Universidad de Valladolid. Vicerrectorado de Docencia. Valladolid. España.

Reque, J.; Bayarri, E.; Sevilla, F, 2013. Diagnóstico selvícola. Universidad de Valladolid. Vicerrectorado de Docencia. PROFOR. Valladolid. España.

Serrada, R., 2003. Regeneración natural; situaciones, concepto, factores y evaluación. Cuad. Soc. Esp. Cien. For. № 15: 11 -15 (2003). Actas de la III Reunión sobre Regeneración Natural del Grupo de Selvicultura. Lourizán.

Serrada, R., 2005. La calidad de las repoblaciones forestales y repoblaciones forestales de calidad. Actas del 4CFE. SECF. Zaragoza.

Serrada, R., 2011. Apuntes de Selvicultura General. FUCOVASA. ETSIM. Madrid.

Serrada, R; Madrigal, A. y González Doncel, I., 1997. Some comments about how the close- 
to-nature silvicultural systems can be implemented in education. Meeting of Silviculture Professors, IUFRO S6.06-04 Education and Research in Silviculture. 1997, Czech Republic. Brno.

Serrada, R.; Montero, M. y Reque, J. (editores). 2008. Compendio de Selvicultura Aplicada en España. INIA y FUCOVASA. Madrid.

Serrada Hierro, R.; Aroca Fernández, M.J.; Roig Gómez, S.; Bravo Fernández, J.A.; Gómez Sanz, V.; 2011. Impactos, vulnerabilidad y adaptación al cambio climático en el sector forestal. Notas sobre gestión adaptativa de las masas forestales ante el cambio climático. Ministerio de Medio Ambiente y Medio Rural y Marino. Madrid. 126 pp. 
